\title{
Non-Alcoholic Steatohepatitis (NASH) - A Review of a Crowded Clinical Landscape, Driven by a Complex Disease
}

Julia M Fraile ${ }^{1,2}$

Soumya Palliyil'

Caroline Barelle ${ }^{2}$

Andrew J Porter ${ }^{1,2}$

Marina Kovaleva ${ }^{2}$

'Scottish Biologics Facility, University of Aberdeen, Aberdeen, AB25 2ZP, UK;

${ }^{2}$ Elasmogen Ltd, Aberdeen, AB25

2ZP, UK
Correspondence: Julia M Fraile; Marina Kovaleva

Elasmogen Ltd, Liberty Building,

Foresterhill Road, Aberdeen, AB25 2ZP,

UK

Tel +44 I 224438574

Email julia.martinezfraile@elasmogen.

com; marina.kovaleva@elasmogen.com

\begin{abstract}
Non-alcoholic steatohepatitis (NASH) is a progressive form of non-alcoholic fatty liver disease (NAFLD), characterized by chronic inflammation and accumulation of fat in liver tissue. Affecting estimated 35 million people globally, NASH is the most common chronic liver condition in Western populations, and with patient numbers growing rapidly, the market for NASH therapy is projected to rise to $\$ 27.2 \mathrm{~B}$ in 2029. Despite this clinical need and attractive commercial opportunity, there are no Food and Drug Administration (FDA)-approved therapies specifically for this disease. Many have tried and unfortunately failed to find a drug, or drug combination, capable of unravelling the complexities of this metabolic condition. At the time of writing this review, only Zydus Cadila's new drug application for Saroglitazar had been approved (2020) for NASH therapy in India. However, it is hoped that this dearth of therapy options will improve as several drug candidates progress through late-stage clinical development. Obeticholic acid (Intercept Pharmaceuticals), Cenicriviroc (Allergan), Aramchol (Galmed Pharmaceuticals), Resmetirom (Madrigal Pharmaceuticals), Dapagliflozin and Semaglutide (Novo Nordisk) are in advanced Phase 3 clinical trials, while Belapectin (Galectin Therapeutics), MSDC0602K (Cirius Therapeutics), Lanifibranor (Inventiva), Efruxifermin (Akero) and Tesamorelin (Theratechnologies) are expected to start Phase 3 trials soon. Here, we have performed an exhaustive review of the current therapeutic landscape for this disease and compared, in some detail, the fortunes of different drug classes (biologics vs small molecules) and target molecules. Given the complex pathophysiology of NASH, the use of drug combination, different mechanisms of actions and the targeting of each stage of the disease will likely be required. Hence, the development of a single therapy for NASH seems challenging and unlikely, despite the plethora of later stage trials due to report. We therefore predict that clinical, patient and company interest in pipeline and next-generation therapies will remain high for some time to come.
\end{abstract}

Keywords: NASH, NAFLD, liver fibrosis, clinical trial

\section{Introduction}

Non-alcoholic steatohepatitis (NASH) is a severe form of non-alcoholic fatty liver disease (NAFLD), characterized by the presence of liver inflammation and hepatocyte injury (ballooning) due to fat accumulation. ${ }^{1}$ Although it develops typically in the absence of excessive alcohol consumption, NAFLD is related to an unhealthy diet and a lack of physical activity. Affecting 35 million people globally, NAFLD is the most common chronic liver condition in Western populations and, with patient 
numbers growing rapidly, the market is expected to rise towards \$27.2 B in 2029. ${ }^{2}$ There are four different clinical phases described for NAFLD. Phase 1 is characterized by simple steatosis and is considered harmless. Some patients progress to Phase 2 developing inflammation and ballooning (NASH). Phase 3 is defined by the presence of NASH with persistent inflammation resulting in liver fibrosis (scarring), which is considered the strongest predictor of liver-related events in NASH patients. Over time, this 3rd stage can lead to a more serious condition, such as liver cirrhosis (Phase 4) or even cancer, where a liver transplant is the only therapy option. In addition to liver-specific pathology, a diagnosis of NASH is also associated with increased cardiometabolic risk and represents the leading cause of death for these patients. ${ }^{3}$ Last year, a group of experts started a new debate on the best terminology for NAFLD and proposed Metabolic Associated Fatty Liver Disease (MAFLD) as a more appropriate term to reflect the heterogeneity of this disease. ${ }^{4}$ Although the incorporation of this new term leads to a change in the diagnostic criteria, it does not affect the prevalence of the condition in the population. ${ }^{5}$ Since no decision regarding which term should be used had been made by the time of writing this revision, we decided to maintain NAFLD in this article.

During the early stages of this disease, patients often show few or non-specific symptoms, such as tiredness, fatigue or abdominal pain, and therefore NASH is often not diagnosed until later stages of disease progression, using invasive techniques such as liver biopsy. ${ }^{1}$ A late onset diagnosis has given NASH the dubious nom de plume of the "Silent Killer". Due to the lack of a cost-effective and minimally invasive diagnostic test, the prevalence of this disease can only be estimated. In a meta-analysis of several studies, the worldwide prevalence of NAFLD was $25.24 \%$. Among this population, biopsy-confirmed NASH was reported in $59.1 \%$ patients. ${ }^{6}$ Even with earlier diagnosis or improved diagnostic options, NASH patients are currently unable to benefit from a portfolio of treatment options that would typically be available to patients suffering from other major disease indications, such as rheumatoid arthritis or some types of cancer. Reduction in liver fat by bariatric surgery has been shown to reverse NASH and fibrosis in severely obese patients. ${ }^{7}$ However, at the time of article submission, there were no Food and Drug Administration (FDA) or European Medicines Agency (EMA) approved NASH-specific drugs, and life-style modifications focused on a healthy diet and exercise were the primary recommendations for patients.
Hopefully, this situation is about to change, as several drug candidates are in late-stage clinical trials. ${ }^{8}$

The histological evidence for a NASH diagnosis is determined by the NAFLD activity score (NAS), which is a composite of steatosis, inflammation and hepatocyte ballooning and represents a measure of disease activity. Once a diagnosis has been made, the rate of disease progression is represented by the NASH Clinical Research Network (CRN) fibrosis score: 0 (none), 1 (mild or moderate), 2 (perisinusoidal fibrosis with portal/ periportal fibrosis), 3 (bridging fibrosis) and 4 (cirrhosis). ${ }^{9}$ Using this fibrosis severity score, the FDA and EMA have encouraged sponsors to focus their drug development activities on those stages of the disease with greatest need, defined as non-cirrhotic NASH with liver fibrosis (fibrosis score greater than 1 but less than 4). Regulators have also highlighted the need to identify less-invasive biomarkers to supplant liver biopsy as the gold standard for diagnosis and assessment of the disease, enabling much broader testing and earlier diagnosis, as well as accelerated drug development outcomes. Furthermore, for approval of Phase 3 clinical studies, sponsors have been recommended to evaluate $\mathrm{NASH}$ and fibrosis independently and to consider the following two primary endpoints: (i) improvement of liver fibrosis greater than or equal to stage 1 (NASH CRN fibrosis score) without worsening of NASH and/or (ii) resolution of NASH (defined by the absence of isolated fatty liver disease or simple steatosis) with no worsening of liver fibrosis. ${ }^{10}$

In this review, we have summarized the complex therapeutic landscape of NAFLD/NASH and compared the different drug classes and pathway targets, focusing on those compounds that are in late-stage clinical development and by definition are better placed to succeed in the "race" for the first FDA-approved and specific therapeutic option against NASH.

\section{NASH (Mono) Therapies in Late-Stage Development}

Saroglitazar Magnesium (Lipaglyn) is a first-in-class therapy already approved in India for the treatment of type 2 diabetes (T2D) and dyslipidemia that acts as a dual peroxisome proliferator-activator receptor (PPAR) alpha and gamma agonist, lowering high blood triglycerides and blood sugar. The PPAR family is composed of three members, PPAR $\alpha$, $\operatorname{PPAR} \gamma$ and PPAR $\delta$, which are ubiquitously expressed in different organs and play an essential role in lipid and glucose metabolism, and inflammation. ${ }^{11}$ Zydus Cadila 
Ltd's new drug application (NDA) for Saroglitazar was accepted in 2020 for NASH treatment in India after achieving positive results in a Phase 3 clinical trial, EVIDENCES II, in Indian NASH patients (Figure 1). After 52 weeks of treatment, the trial successfully met primary and secondary endpoints, demonstrating histological improvement of NASH by liver biopsy. In another Phase 2 clinical trial, EVIDENCES I, Saroglitazar showed improvement measured as a reduction in liver enzymes and lipid values in NAFLD patients. Outside India, a Phase 2 EVIDENCES IV trial in the United States (ClinicalTrials.gov NCT03061721) completed in October 2020 and met its primary endpoint of reduced ALT in NAFLD and NASH patients after 16 weeks of treatment with Saroglitazar. ${ }^{12}$

Obeticholic acid (Ocaliva) is a semisynthetic analog of the bile acid, chenodeoxycholic acid, and acts as a Farnesoid $\mathrm{X}$ receptor (FXR) agonist (Figure 2). FXR is a member of the nuclear receptor superfamily that regulates a variety of genes involved in bile acid synthesis and transport, and in glucose and lipid metabolism. ${ }^{13}$ Already approved for the treatment of primary biliary cholangitis (PBC), Obeticholic acid is being developed by Intercept Pharmaceuticals Inc and was considered to be "leading the field" until the FDA rejected the company's NDA for NASH treatment in June 2020. Intercept's NDA application was based on an interim analysis from their 18-month Phase 3
REGENERATE (ClinicalTrials.gov NCT02548351) clinical trial of Obeticholic acid with nearly 2500 NASH patients with fibrosis enrolled (Figure 1). ${ }^{14}$ The study demonstrated an improvement of liver fibrosis without worsening of NASH as one of the two endpoints that the FDA requires for securing approval. However, the regulatory agency has asked the company to provide longer term data from the Phase 3 REGENERATE clinical study with specific reference to efficacy and safety and declined to support an accelerated approval for this medication. The FDA also reported several active liver toxicity signals associated with Obeticholic acid treatment, not already mentioned on its current drug label. In addition, an increase in low-density lipoprotein cholesterol and total cholesterol has been observed after treatment with Obeticholic acid in the Phase 2 FLINT clinical trial (ClinicalTrials.gov NCT01265498). ${ }^{15}$ Undeterred by this initial set-back, Intercept Pharmaceuticals are also sponsoring another Phase 3 REVERSE clinical trial (ClinicalTrials.gov NCT03439254) to evaluate the efficacy and safety of Obeticholic acid in patients with compensated cirrhosis due to NASH.

Although Obeticholic acid had been heralded as the most advanced drug for the treatment of NASH, the FDA response to their NDA, together with the potential for liver toxicity problems, was encouraging enough for Intercept's competitors to continue playing their part in this drug race.
F1

\begin{tabular}{|c|c|c|}
\hline NAFLD & Early NASH & Advanced NASH \\
\hline & Zydus Cadila Saroglitaza & $\begin{array}{l}\text { PPARa/NDA approved } \\
\text { PPARy in India }\end{array}$ \\
\hline & Intercept & Obeticholic acid \\
\hline \multirow[t]{5}{*}{ Madrigal Pharmaceuticals } & Resmetirom & THR $\beta$ \\
\hline & & $\begin{array}{ll}\text { Allergan } & \text { Cenicriviroc } \\
\text { CCR2/CCR5 }\end{array}$ \\
\hline & & $\begin{array}{l}\text { Galmed Aramchol } \\
\text { Pharmaceuticals }\end{array}$ \\
\hline & Dapagliflozin & SGLT2 \\
\hline & & $\begin{array}{lc}\text { Novo } & \text { Semaglutide } \\
\text { Nordisk } & \text { GLP-1R }\end{array}$ \\
\hline
\end{tabular}

Figure I Phase 3 clinical therapies for NASH treatment aligned to different stages of the disease. NASH progression is represented by the NASH CRN fibrosis score: NAFLD (none), FI (mild or moderate), F2 (perisinusoidal fibrosis with portal/periportal fibrosis), F3 (bridging fibrosis) and F4 (cirrhosis). Company, drug name and targets are indicated for each clinical therapy.

Abbreviations: PPAR, peroxisome proliferator-activator receptor; FXR, Farnesoid X receptor; THR $\beta$, thyroid hormone receptor-beta; CCR2/CCR5, chemokine 2 and 5 receptor; SCDI, stearoyl-coenzyme A desaturase-I; SGLT2, sodium-glucose co-transporter-2; GLP-IR, glucagon-like peptide I receptor. 


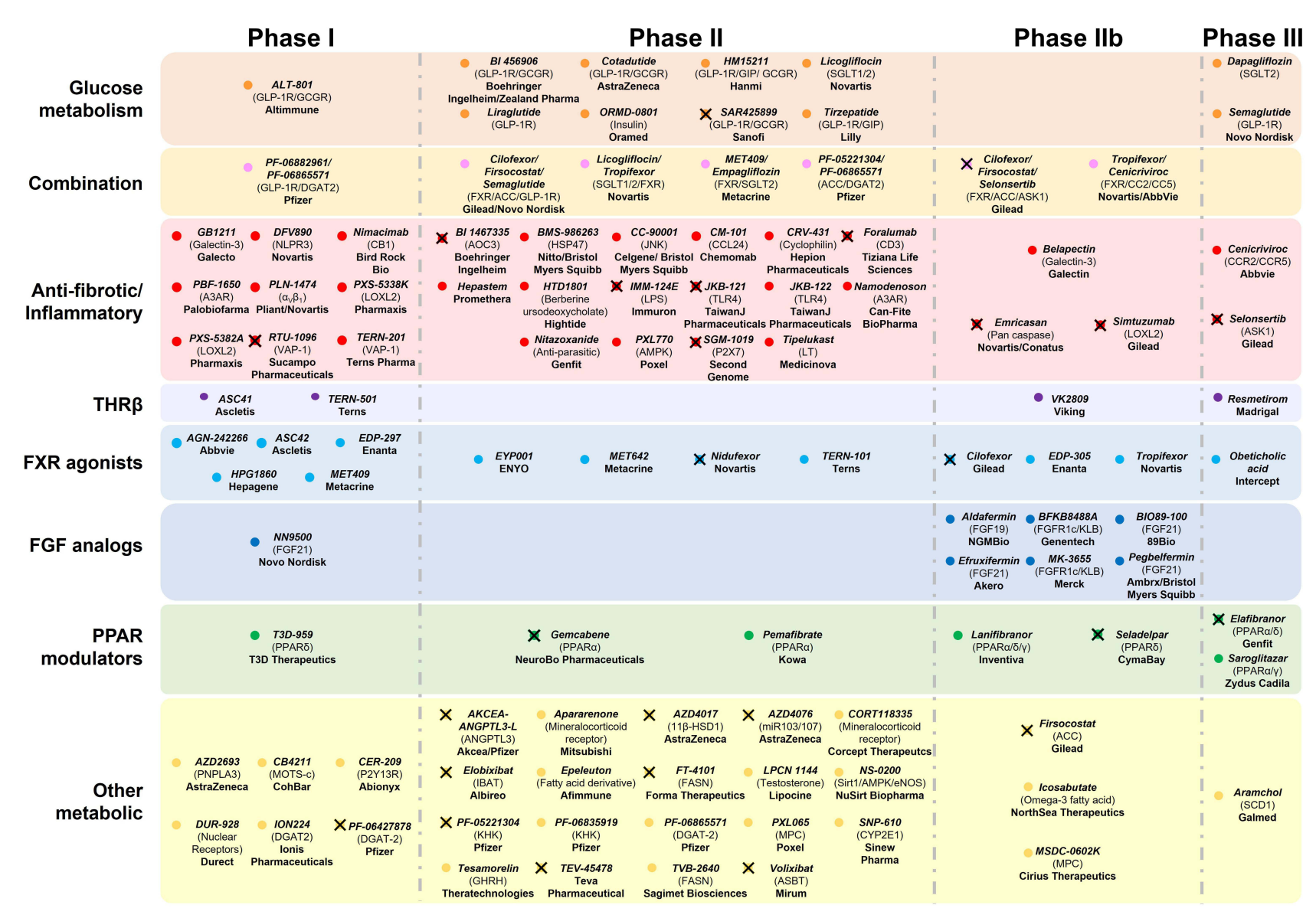

Figure 2 Therapeutic landscape for NAFLD/NASH with targeted pathways. Current clinical therapies for NASH are grouped into different target classes and clinical development stages. A cross indicates that the drug has been discontinued for NASH therapy from the company's pipeline.

Abbreviations: THR $\beta$, thyroid hormone receptor-beta; FXR, Farnesoid X receptor; FGF, fibroblast growth factor; PPAR, peroxisome proliferator-activator receptor.

Indeed, Intercept Pharmaceuticals themselves have developed another FXR agonist for NASH treatment, INT-787, which, whilst still in preclinical studies, is believed to be more selective than Obeticholic acid. The company plans to start clinical trials with INT-787 in 2021.

Resmetirom, or MGL-3196, is a first-in-class oral thyroid hormone receptor-beta (THR $\beta$ ) agonist developed by Madrigal Pharmaceuticals Inc (Figure 2). THR $\beta$ is the main receptor for thyroid hormones in the liver and plays an essential role in lipid metabolism. ${ }^{16}$ Phase 2 clinical data showed that Resmetirom treatment resulted in a significant reduction in hepatic fat in patients with $\mathrm{NASH},{ }^{17}$ and supported its advancement into Phase 3 clinical development (Figure 1). Madrigal Pharmaceuticals is currently sponsoring two Phase 3 studies: MAESTRO-NASH (ClinicalTrials.gov NCT03900429) and MAESTRO-NAFLD-1 (ClinicalTrials. gov NCT04197479), comprising patients at different stages of the disease. MAESTRO-NASH began in 2019 and is expected to enroll up to $2000 \mathrm{NASH}$ patients with fibrosis (CRN stage 2 or 3). This trial is divided into two different arms. Initially, 900 patients will be enrolled with the primary endpoint of NASH resolution after 52 weeks of treatment, with at least a 2-point reduction in NAS and no worsening of fibrosis. Additional 1100 patients will be added to the study after the first year of treatment to evaluate the reduction in liver-related adverse events or progression to cirrhosis for up to further 54 months. Early data demonstrated liver fat reduction after three months of treatment with Resmetirom and was also shown to be a predictor for NASH resolution and fibrosis reduction in subsequent liver biopsy. Madrigal's second Phase 3 study, MAESTRO-NAFLD-1, was initiated at the end of 2019 and is expected to enroll 700 patients with early stages of the disease (NAFLD or presumed NASH). Unlike MAESTRO-NASH trial, MAESTRO-NAFLD-1 is a nonbiopsy study and its primary endpoint is to evaluate the effect of Resmetirom on the incidence of adverse effects, compared to placebo. The company expects to release data from this study by the end of 2021. Separate from its benefits in liverrelated conditions, Madrigal Pharmaceuticals hopes to also highlight Resmetirom's potential to decrease the elevated 
cardiovascular risk seen in NASH patients, through a reduction in heart attacks and strokes both during the trials and patient follow-up.

Cenicriviroc (CVC), is a novel, orally administered, potent, small molecule agonist that acts to block chemokine 2 and 5 receptors (CCR2/CCR5), both with wellknown roles in liver inflammation and fibrosis (Figure 2). ${ }^{18} \mathrm{CVC}$ has been developed by Allergan Inc (an AbbVie Inc company) and has received a Fast Track designation by the FDA for the treatment of NASH. Although CVC treatment failed to meet its primary endpoint in the Phase 2b CENTAUR study (ClinicalTrials. gov NCT02217475) defined as histological improvement in NAS without worsening of fibrosis, ${ }^{19}$ it did improve levels of measurable liver fibrosis without worsening NASH. This data provided enough confidence for the company to continue to evaluate the efficacy and safety of this drug in a Phase 3 AURORA trial (Figure 1) (ClinicalTrials.gov NCT03028740), enrolling approximately $2000 \mathrm{NASH}$ patients with fibrosis (CRN stage 2 or 3$).{ }^{20}$ Like others in this space, this study was thought to be performed in two arms: (i) lasting approximately one year to examine the improvement in liver fibrosis by at least 1 stage with no worsening of NASH in patients and (ii) lasting further 7 years to analyze long-term clinical outcomes by following histopathologic progression to cirrhosis, other liver-related outcomes and all-cause mortality in patients with stage 3 fibrosis. However, at the beginning of 2021 the AURORA phase 3 clinical trial terminated due to lack of efficacy on the results of the first part of the study. The company has not indicated future plans for CVC yet, but the termination of the AURORA study sows a seed of doubt about its efficacy as a monotherapy. Allergan is also conducting a 48-week Phase 2b TANDEM study (ClinicalTrials.gov NCT03517540) in collaboration with Novartis AG to evaluate the safety and efficacy of a combination of Tropifexor, a FXR agonist, and CVC in 200 patients with biopsy-proven NASH with fibrosis (CRN stage 2 or 3) (Figure 2). ${ }^{21}$

Aramchol is a first-in-class, once-daily, oral stearoylcoenzyme A desaturase-1 (SCD1) modulator developed by Galmed Pharmaceuticals Ltd and granted FDA Fast Track designation status for the treatment of NASH. SCD1 is considered a mediator of liver steatosis and fibrosis because of its role in fatty acid biosynthesis. ${ }^{22,23}$ In 2018, the company published the results of their Phase $2 \mathrm{~b}$ ARREST clinical trial (ClinicalTrials.gov
NCT02279524) that documented Aramchol treatment in 247 NASH patients who were all clinically overweight or obese and had prediabetes or T2D. This one-year study demonstrated liver fat reduction, biochemical improvement and both NASH and fibrosis resolution, with favorable safety and tolerability profiles. Buoyed by these results, Galmed Pharmaceuticals started, one year later, their 52-week Phase 3/4 ARMOR study (ClinicalTrials. gov NCT04104321) with NASH patients with advanced fibrosis (CRN stage 2 or 3 ) to evaluate the efficacy of Aramchol on NASH resolution, fibrosis improvement and a series of clinical outcomes related to NASH progression (Figure 1). Primary completion of this study is estimated in 2022 .

Galectin Therapeutics Inc, Cirius Therapeutics Inc, Inventiva Pharma SA, Akero Therapeutics Inc and Theratechnologies Inc, are also moving their NASH therapeutic candidates into later stage clinical development, encouraged by patient outcomes, safety profiles and the hitting of key endpoints in early clinical studies.

Galectin Therapeutics has started recruiting NASH patients with compensated cirrhosis for a Phase $2 b / 3$ NAVIGATE clinical study (ClinicalTrials.gov NCT04365868) to begin evaluating the safety and efficacy of Belapectin (GR-MD-02), a complex carbohydrate that targets Galectin-3, a $\beta$-galactoside-binding lectin that plays an important role in inflammatory responses, ${ }^{24}$ for the prevention of esophageal varices. The company already reported in their Phase $2 \mathrm{~b}$ NASH-CX clinical trial (ClinicalTrials.gov NCT02462967) that Belapectin treatment, when compared to the placebo arm, safely reduced the hepatic venous pressure gradient in NASH cirrhosis patients without esophageal varices. $^{25}$ Galectin Therapeutics' next NAVIGATE study will consist of an 18-month Phase $2 \mathrm{~b}$ extension study followed by an interim analysis to consider any adaptive treatment modifications before a final evaluation in an 18-month Phase 3 trial. Endpoints will assess the proportion of patients who develop new esophageal varices after 18 months of Belapectin treatment compared to placebo (primary) and the effect of this treatment on the incidence of long-term clinically significant cirrhosis-related events (key secondary endpoint).

Cirius Therapeutics is another company planning to enter Phase 3 development for NASH treatment with its drug MSDC-0602K. This second-generation thiazolidinedione is designed to modulate the mitochondrial pyruvate carrier (MPC), a protein complex that regulates the entry 
of pyruvate into the mitochondria, with minimum activation of PPAR $\gamma$ (Figure 2). ${ }^{26}$ The company demonstrated in their 12-month Phase 2b EMMINENCE clinical trial (ClinicalTrials.gov NCT02784444), involving over 400 subjects with biopsy proven NASH (CRN stage 2 or 3), that treatment with MSDC-0602K significantly improved glycemic control and liver enzyme levels compared to the placebo arm. Furthermore, MSDC-0602K treatment was well tolerated and resulted in a dose-dependent improvement in liver histopathology, which, although not being statistically significant, supported the continuation to Phase 3 MMONARCh-1 clinical development in NAFLD/NASH patients with T2D (ClinicalTrials.gov NCT03970031).

Lanifibranor is an oral small molecule that activates all three PPAR isoforms (PPAR $\alpha$, PPAR $\delta$ and PPAR $\gamma$ ), inducing anti-fibrotic, anti-inflammatory and other beneficial metabolic changes in the body, and delivers these outcomes by decreasing triglyceride levels and increasing high-density lipoprotein cholesterol levels and insulin sensitization (Figure 2). Developed by Inventiva, it is the only pan-PPAR agonist in clinical development for NASH. The company completed a 24-week Phase 2b NATIVE clinical trial (ClinicalTrials.gov NCT03008070) and demonstrated that the higher dose used in this study (1200 mg per day) reduced by at least two points the steatosis activity fibrosis (SAF) score (SAF is a measure that combines the degree of hepatocellular inflammation and cellular ballooning) with no worsening of fibrosis (primary endpoint). The study also met secondary endpoints with the same dose, which included resolution of NASH with no worsening of fibrosis and improvement of fibrosis without worsening of NASH. Inventiva was pleased to be able to highlight that Lanifibranor is the only candidate drug to achieve statistically significant results for both of the primary endpoints specified by the FDA and the EMA, a key deliverable for those companies and drugs seeking accelerated approval during Phase 3 development. The FDA responded to this strong $2 \mathrm{~b}$ data set by indicating that a single pivotal Phase 3 study could be sufficient to support the filing of an NDA in the United States. This feedback from regulators, together with Breakthrough Therapy designation in NASH, encouraged the initiation of a Phase 3 NATIVE3 pivotal clinical trial with Lanifibranor, which is expected to recruit its first patients sometime in 2021.

Efruxifermin (EFX), formerly AKR-001, is an analogue fusion protein of FGF21, which is a member of the fibroblast growth factor family that regulates multiple metabolic pathways and cellular processes, ${ }^{27}$ and was "designed" by Akero to increase insulin sensitivity, improve lipoproteins, reduce liver fat and inflammation and reverse levels of fibrosis (Figure 2). The decision to continue EFX development in 2020 was taken after positive histological results demonstrated a reduction in liver fat and improvement in liver fibrosis, obtained in a 16week Phase 2a BALANCED study in NASH patients. ${ }^{28}$ Akero now intends to evaluate the efficacy of EFX in NASH patients in a Phase $2 \mathrm{~b} / 3$ adaptive clinical trial. This two-part study will be composed of a 24-week Phase $2 b$ HARMONY trial that has already started and is designed to select an appropriate patient dose, which will then be used to inform and further evaluate their drug in a more expansive Phase 3 study.

Tesamorelin is an FDA-approved medication for the treatment of HIV-associated lipodystrophy developed by Theratechnologies. Tesamorelin is a synthetic form of growth-hormone-releasing hormone (GHRH), which participates in a wide range of physiological pathways, including the stimulation of the growth hormone secretion from the pituitary gland. ${ }^{29}$ Data presented by the company from a 12 month clinical trial evaluating the effect of Tesamorelin on liver fat and steatohepatitis in HIV-infected NAFLD patients (ClinicalTrials.gov NCT02196831) demonstrated that participants treated with this compound had a greater reduction in hepatic fat fraction compared to those receiving placebo (Figure 2). ${ }^{30}$ In December 2020, the FDA approved the continuation of Tesamorelin to Phase 3 development for NASH treatment and recommended a meeting with the company to discuss the details of the proposed trial design. The study is expected to start in Q3 2021 and will enroll patients with liver-biopsy confirmed NASH (CRN stage 2 or 3 ). After 18 months of treatment with Tesamorelin or placebo, a second liver biopsy will be performed and NASH resolution with no worsening of fibrosis or fibrosis improvement $\geq 1$ stage with no worsening of NASH will be compared between both groups (primary endpoints). Theratechnologies is planning its strategy to get approval from the EMA to initiate a Phase 3 clinical trial of Tesamorelin for NASH treatment also in Europe.

\section{Anti-Diabetic Drugs for NASH Treatment}

NAFLD is closely associated with $\mathrm{T} 2 \mathrm{D},{ }^{31}$ but despite this established clinical link there is currently no go-to pharmacotherapy for NASH patients with T2D. Pioglitazone 
(Actos), a PPAR $\gamma$ agonist developed by Takeda Pharmaceutical Company Limited, is the only recommended medication for patients with both pathologies. However, the FDA and European Association for the Study of the Liver (EASL) have voiced several safety concerns that have prevented any enthusiastic support for its wide clinical use. Pioglitazone in combination with vitamin $\mathrm{E}$ improved liver histology in patients with NASH and T2D (ClinicalTrials.gov NCT01002547). ${ }^{32}$ In this regard, a Phase 3 PIVENS study (ClinicalTrials.gov NCT00063622) demonstrated that treatment with vitamin E showed better results than placebo in improving liver histology in NASH patients without diabetes. ${ }^{33}$ Although the EASL guideline states that vitamin E may be used in non-cirrhotic and non-diabetic NASH patients, they advise that further studies are required for firm recommendations to be made concerning extending vitamin $\mathrm{E}$ usefulness.

Currently, several new diabetic agents are in clinical trials for NASH treatment, including sodium-glucose cotransporter-1/2 (SGLT1/2) inhibitors and glucagon-like peptide 1 receptor (GLP-1R) agonists (Figure 2). ${ }^{34}$ Ahead of these newer agents, Dapagliflozin, Liraglutide, Dulaglutide and Semaglutide are examples of already approved anti-diabetic therapies that are now under latestage consideration for NASH. Of those molecules that appear to be progressing well, Dapagliflozin, developed by Bristol-Myers Squibb and AstraZeneca Ltd, is currently in Phase 3 and is an inhibitor of SGLT2, which in turn impedes glucose reabsorption in the proximal tubule leading to glucosuria and plasma glucose reduction (Figure 1). The Dapagliflozin DEAN study (ClinicalTrials.gov NCT03723252) is still recruiting and will evaluate its safety and efficacy on NASH histological improvement in approximately 100 participants.

Semaglutide, developed by Novo Nordisk A/S, is approved for the treatment of T2D, functions as an agonist of GLP-1R and plays an essential role in insulin secretion (Figure 2). ${ }^{34}$ A 72-week Phase 2 clinical study was performed using Semaglutide to evaluate the efficacy and safety of three different doses of subcutaneous administration versus placebo in 320 subjects with biopsy-confirmed NASH and liver fibrosis stages 1, 2 or 3 (ClinicalTrials. gov NCT02970942). For this study: (i) NASH resolution without worsening of fibrosis and (ii) improvement of at least one fibrosis stage with no worsening of NASH were defined as primary and secondary endpoints. Only patients with stage 2 or 3 fibrosis levels were assessed in this cohort (230 individuals). A fuller range of analyses were performed on all 320 patients and indicated that the higher dose of Semaglutide $(0.4 \mathrm{mg}$ per day) resulted in a significantly higher percentage of patients with NASH showing resolution of the condition with no worsening of fibrosis when compared to placebo. It is also worth noting, however, that no significant differences were observed between the different doses used and the percentage of patients with an improvement in fibrosis staging, including the placebo control. ${ }^{35}$ The company has recently started recruiting patients with non-cirrhotic NASH in a Phase 3 clinical trial (ClinicalTrials.gov NCT04822181) that is expected to last for approximately 5 years (Figure 1). In addition, Novo Nordisk and Gilead Sciences Inc have recently completed an extensive Phase 2a clinical trial (ClinicalTrials.gov NCT03987074) that evaluates the safety, tolerability, and efficacy of Semaglutide alone or in combination with Cilofexor (FXR agonist) and/or Firsocostat (ACC inhibitor), in more than $100 \mathrm{NASH}$ patients with a fibrosis score of 2 or 3 (Figure 2). All combinations were well tolerated, and a statistically significant improvement in hepatic steatosis and liver injury was observed in the combination arms compared to Semaglutide alone (at 24 weeks in post-hoc analysis). Novo Nordisk and Gilead have planned a further Phase $2 \mathrm{~b}$ study beginning in the second half of 2021 to analyze the effect of Semaglutide alone and in combination with Cilofexor and/or Firsocostat on liver fibrosis improvement and NASH resolution in 440 NASH patients with compensated cirrhosis.

\section{Discontinued Therapeutics for NASH}

Over the last few years, several pharmaceutical companies have failed to find a drug for the treatment of NASH typically because of a lack of efficacy, toxicity or elements of both. Two higher-profile medications that were discontinued after failing Phase 3 clinical trials were Selonsertib (Gilead) and Elafibranor (Genfit SA). Selonsertib is an oral small molecule inhibitor of the apoptosis signal-regulating kinase 1 (ASK1) and eventually failed in two different Phase 3 trials, STELLAR-3 (ClinicalTrials.gov NCT03053050) and STELLAR-4 (ClinicalTrials.gov NCT03053063). Both studies enrolled over 1600 patients in total and were designed to evaluate the safety and efficacy of Selonsertib when used to treat NASH patients with stage 3 fibrosis (STELLAR-3) or compensated cirrhosis, stage 4 fibrosis (STELLAR-4). Although Selonsertib was generally well tolerated with no 
safety concerns, none of the studies achieved their primary endpoint of an improvement in fibrosis without worsening of NASH (after 48 weeks of treatment). ${ }^{36}$ Gilead also tested Selonsertib as part of a combination therapy with Firsocostat and Cilofexor in a Phase 2b ATLAS clinical trial (ClinicalTrials.gov NCT03449446) with NASH patients with advanced fibrosis (CRN stage 3 or 4). Again, all treatments, either alone or in dual combination, were well tolerated but none met their primary endpoint of reducing fibrosis without worsening of NASH. However, improvements in multiple secondary endpoints involving measurements of fibrosis and liver function were observed in patients treated with both Firsocostat and Cilofexor, when compared with the placebo group. ${ }^{37}$

Until recently, Genfit was considered the most advanced competitor to Intercept Pharmaceuticals Obeticholic acid, with its own oral treatment, Elafibranor, which acts simultaneously on nuclear receptors shown to play critical roles in the development of NASH, PPAR $\alpha$ and PPAR $\delta$. Unlike other small molecules targeting PPARs, Elafibranor did not demonstrate the adverse effects commonly associated with PPAR $\gamma$ activation, with good safety and tolerability profiles in patients. In Genfit's large international Phase $2 b$ trial, Elafibranor delivered NASH resolution without worsening of fibrosis and improved the metabolism of triglycerides and lipids in patients with advanced disease. ${ }^{38}$ This success encouraged the company to design a 72-week Phase 3 RESOLVE-IT clinical trial (ClinicalTrials.gov NCT02704403) to evaluate the efficacy and safety of Elafibranor, compared to placebo, in more than 1000 patients with NASH and fibrosis. Unfortunately, Genfit was disappointed to report in May 2020 that the study did not meet its key primary endpoints. Although both safety and tolerability profiles of Elafibranor were good, only $19.2 \%$ of the patients who received this drug achieved NASH resolution without worsening of fibrosis, compared to $14.7 \%$ of patients in the placebo arm. For fibrosis, the results were even starker, with $24.5 \%$ of Elafibranor-treated patients showing fibrosis improvement of at least one stage compared to an almost comparable $22.4 \%$ of patients in the placebo arm. No statistically significant differences between Elafibranor and placebo groups were found in these or any other metabolic parameters. Although Genfit will continue to develop Elafibranor for the treatment of $\mathrm{PBC}$, the company has decided to terminate its Phase 3 RESOLVE-IT trial. Genfit has not abandoned liver disease completely as they are still committed to testing the antifibrotic properties of the repurposed Nitazoxanide, originally considered an anti-parasitic drug, and are now in a Phase 2 study with patients with NASH-induced stage 2 or 3 staging of fibrosis (Figure 2). In addition, Genfit is seeking regulatory approval for a new diagnosis and disease management technology, NIS4TM, which claims to be a noninvasive test capable of identifying patients at highest risk of liver disease, based on the quantification of specific biomarkers present in their blood when considered in combination with a series of, patient specific, metabolic risk factors.

\section{Antibodies in Development for NASH}

Most treatments for NASH that have made it all the way to clinical trials are based on the use of small molecules, typically inhibitors or agonists. In other disease areas such as inflammation or oncology, monoclonal antibodies (mAbs) have slowly begun to dominate as the "go-to" class for drug therapy with drugs such as Humira ${ }^{\circledR}$ selling in excess of $\$ 100 \mathrm{~B}$ during its patent protected lifespan. However, for the treatment of metabolic diseases such as $\mathrm{NASH}$, the jury is still out on the ability or potency of mAbs to have the same impact on disease progression. To date, only a few mAb therapies have made it all the way to clinical studies (Table 1). The efficacy of Simtuzumab, a monoclonal antibody developed by Gilead and directed against lysyl oxidase-like-2 (LOXL2), an amine oxidase that promotes liver fibrosis by driving covalent crosslinking of collagen fibers, ${ }^{39,40}$ was evaluated in two Phase $2 b$ clinical trials involving patients with advanced NASH. Unfortunately, both studies were stopped after 96 weeks due to a lack of efficacy. ${ }^{41}$ Tiziana Life Sciences Plc terminated their Phase 2 NASH study, designed to evaluate the efficacy of Foralumab. This orally administered $\mathrm{mAb}$ recognized $\mathrm{CD} 3$, a member of the immunoglobulin superfamily, which acts as a mediator for signal transduction and is expressed by a high-percentage of circulating peripheral $\mathrm{T}$ cells. ${ }^{42}$ Another orally delivered $\mathrm{mAb}$ that failed to meet its clinical end points for NASH was IMM-124E. Developed by Immuron Ltd, this polyclonal antibody mixture was specific to lipopolysaccharide (LPS) and other pathogenic bacterial components in the human gastrointestinal tract. Data from its Phase 2 clinical trial (ClinicalTrials.gov NCT02316717) showed that treatment with IMM-124E did not produce any evidence of clinical benefit and was not able to reduce the fat content of the liver in NASH patients, but it did decrease serum LPS 
Table I Antibodies in Clinical Development for the Treatment of NASH

\begin{tabular}{|l|c|c|c|c|}
\hline Antibody & Company & Target & Clinical Phase & Status \\
\hline Simtuzumab & Gilead & LOXL2 & $2 b$ & Discontinued \\
Foralumab & Tiziana Life Sciences & CD3 & 2 & Discontinued \\
IMM-I24E & Immuron & LPS & 2 & Discontinued \\
BFKB8488A & Genentech & FGFRIC/KLB & $2 b$ & Recruiting \\
MK-3655 & Merck & FGFRIc/KLB & $2 b$ & Recruiting \\
CM-I0I & Chemomab & CCL24 & $2 \mathrm{a}$ & Recruiting \\
Nimacimab & Bird Rock Bio & CBI & Ib & Completed \\
\hline
\end{tabular}

levels, as well as two biomarkers associated with liver function (AST, ALT). The end came for IMM-124E when negative results were also reported in a second Phase 2 clinical study involving pediatric NAFLD patients (ClinicalTrials.gov NCT03042767). This study also failed to meet its primary endpoint, showing no substantial changes in ALT levels when compared to the placebo control arm.

The flexibility and potency of $\mathrm{mAb}$ drugs continue to be improved with format refinements often originally made as treatments for oncology, eventually being adopted by drug-discovery teams tackling other disease classes. One such refinement or improvement is the ability of a single $\mathrm{mAb}$ (or mAb-related drug format) to recognize and bind two different drug targets. This bispecific capability can aid therapeutic potency, specificity and reduce off-target side effects. BFKB8488A (Genentech Inc) is a full-length, humanized bispecific antiFGFR1c/KLB agonist antibody that selectively activates FGFR1 in a KLB-dependent manner and mimics the actions of FGF21. ${ }^{43}$ Genentech has already demonstrated in a Phase 1b clinical trial (ClinicalTrials.gov NCT03060538) that the subcutaneous injection of BFKB8488A was safe and adequately tolerated, with nausea as the most significant adverse effect. Early indicators of efficacy were also reported with improved markers of cardiometabolic and liver health observed in patients with both T2D and NAFLD, with a dose- and exposure-dependent reduction of liver fat in the NAFLD cohorts in particular. ${ }^{43}$ Encouraged by these results, Genentech has recently initiated a Phase 2b BANFF clinical trial (ClinicalTrials.gov NCT04171765) to evaluate the efficacy, safety and pharmacokinetics of BFKB8488A in NASH patients with a fibrosis score between 2 and 3 (Figure 2 and Table 1).
NGM313 is a monoclonal antibody, originally developed by NGM Biopharmaceuticals Inc, that selectively activates FGFR1c/KLB by binding to a unique epitope of KLB and acts as an insulin sensitizer. NGMBio's Phase $1 \mathrm{~b}$ clinical study demonstrated that a single subcutaneous dose of this antibody significantly reduced liver fat content and improved multiple metabolic parameters in obese, insulin-resistant subjects with NAFLD. Merck \& Co Inc in-licensed NMG313 in 2019 (renamed MK-3655) and has recently started recruiting pre-cirrhotic NASH patients, with or without diabetes, into a Phase $2 b$ clinical study to analyze MK-3655 effects on NASH resolution without worsening of fibrosis, after 52 weeks of treatment (Figure 2 and Table 1). Hopefully, these "next-generation" mAbs from Genentech and Merck with boosted specificity and potency will succeed where others have failed, and we may also see the first-approved antibodies for the treatment of metabolic diseases such as NASH.

Chemomab is developing CM-101, a blocking mAb that targets CCL24, which is a chemokine that modulates inflammatory and fibrotic processes through its receptor CCR3. ${ }^{44} \mathrm{CM}-101$ was tested in a 12 -week Phase $1 \mathrm{~b}$ clinical trial to evaluate its safety, tolerability, pharmacokinetics and pharmacodynamics in NAFLD patients with a follow-up study planned in a similar cohort of NASH patients. Results communicated by the company in January 2021 demonstrated that treatment with CM-101 decreased serum biomarkers of fibrosis and inflammation with no issues with safety or tolerability. Chemomab has recently started enrolling patients in a Phase 2a SPLASH clinical trial that will evaluate the effects of CM-101 in 40 NASH patients with fibrosis stage 2 or 3 (Figure 2 and Table 1). Data from this study are expected in H1 2022.

Finally, Nimacimab, also known as RYI-018 or JNJ2463 , is another antibody at a much earlier clinical stage of development (Figure 2 and Table 1). Nimacimab (Bird Rock Bio Inc) is an antagonist antibody with potentially 
anti-fibrotic, anti-inflammatory and a beneficial metabolic mechanism of action. Nimacimab targets the cannabinoid receptor 1 (CB1), a G-protein coupled receptor, which has been reported to play a role in hepatic inflammation and fibrosis. ${ }^{45}$ Bird Rock Bio successfully completed a Phase $1 \mathrm{~b}$ clinical trial that evaluated the safety, tolerability and pharmacokinetics of their antibody in NAFLD patients and their intention to conduct a Phase $2 \mathrm{~b}$ clinical study has been confirmed.

At a much earlier point in their development (late stage pre-clinical) than any of the studies described above, companies such as Elasmogen Ltd are using the power and flexibility of their soloMER drug discovery platforms to produce smaller antibody-like biologics for the treatment of later stage liver disease, and fibrosis in particular. Whilst the details of this program remain confidential, the use of biand tri-specific formats has been disclosed, including the use of binding domains that employ receptor-mediated internalization to enter cells, facilitating the delivery of small molecules conjugated to their biologic targeting domains. This strategy delivers the promise of increasing potency still further through the combination of biologic and small molecule drugs as a single targeted therapy.

\section{Multiple Points of Intervention - Which Drug-Target Class is "Winning" \\ Possible Winners - Going It Alone}

NAFLD is a complicated, heterogeneous disease, with multiple possible points of therapeutic intervention. In general, therapeutic candidates currently in the clinic are focused on modulating metabolic pathways, reducing inflammation and/or fibrosis. ${ }^{46}$ PPAR agonists represent a targeted therapy that reduces steatosis and inflammation. Several drugs that modulate the activity of these nuclear receptors have been investigated as treatments for $\mathrm{NASH}$ in patients (Figure 2). Two of the most advanced, Elafibranor (Genfit) and Seladelpar (CymaBay Therapeutics Inc), did not meet their primary endpoints, were discontinued and together throw into question the whole approach of using PPAR agonists as a NASH treatment class. However, Inventiva has recently caused a reevaluation of the field by reporting positive results (June 2020) from their Phase 2 NATIVE trial with Lanifibranor, a pan-PPAR agonist.

FXR ligands are a second class of molecules also capable of reducing the levels of both inflammation and fibrosis in patient trials. Several FXR-activating medications are currently under clinical development, each with slightly different structures and pharmacodynamic effects (Figure 2). The most promising, with a clinically demonstrated positive effect on hepatic metabolism, is Obeticholic acid. Unfortunately, Intercept Pharmaceuticals, the drug's developers, received a less than positive response from the FDA in June 2020 with potential liver toxicity flagged as a possible barrier to approval and a request for further clinical data issued. FGF analogs, closely related to FXR agonists, are also being evaluated as a potential NASH therapy. So far, treatment with several FGF19 and FGF21 agonists (both small molecules and bi-specific mAbs) has shown improvement in several metabolic parameters and a reduction in liver fat (Figure 2). Thus, Efruxifermin (Akero), Pegbelfermin (Ambrx Inc/Bristol Myers Squibb), BFKB8488A (Genentech), Aldafermin (NGM Biopharmaceuticals) and MK-3655 (Merck \& Co) are currently being studied in Phase $2 \mathrm{~b}$ development (Figure 2). It is noteworthy that Aldafermin has recently failed a Phase 2 b ALPINE 2/3 study for NASH patients with stage 2 or 3 of liver fibrosis, but NGM Biopharmaceuticals are still evaluating this drug in a Phase $2 \mathrm{~b}$ ALPINE 4 trial in NASH patients with severe fibrosis and compensated fibrosis. Within this target class, there is a secondary race happening between mAbs and small molecule inhibitors. In other disease areas, $\mathrm{mAbs}$ have been shown to deliver improvements in specificity (and potency), safety profile, and dosing frequency (4-week serum half-life), and whilst clinical development times can be shorter than small molecules, mAbs are also large, complex and more costly to manufacture at scale, especially where newer $\mathrm{mAb}$ formats prove to be the candidate molecule of choice.

Antagonistic drugs, such as VK2809 (Viking Therapeutics Inc) and Resmetirom (Madrigal Pharmaceuticals), targeting liver THR $\beta$ are gaining clinical traction. Now in Phase 2b and Phase 3, respectively, both have reported positive results, especially the reduction of lipids. Currently, in a class on its own, Aramchol (Galmed) has a unique mechanism of action, modulating the activity of SCD1. Although this is a target that other companies have yet to fall behind, the data so far suggest that this strategy is capable of reducing key metabolic markers of NASH and even fibrosis staging levels.

Whilst much of this review has focused on the treatment of earlier stage disease, the lack of reliable diagnostic tests (currently biopsy remains the "gold-standard") means that patients continue to present with late-stage disease. Therefore, anti-fibrotic therapies targeting stellate cell 
activation or extracellular matrix production will still be required for some time to come. Allergan will need to indicate future plans for CVC after the termination of the Phase 3 AURORA trial, Galectin Therapeutics is currently evaluating Belapectin in a Phase $2 \mathrm{~b}$ clinical study and new entrants, such as Elasmogen Ltd with their soloMER platform, are also developing anti-fibrotic/anti-stellate therapies.

\section{Co-Therapies - Working Together to Win the Race}

Although most NASH clinical trials are based on a single agent (mono) therapy, the complex pathophysiology of this disease and the multiple often redundant "escape" pathways to therapy, strongly suggest that the development of a single drug capable of effectively treating most patients is becoming increasingly challenging and unlikely. ${ }^{47}$ Hence, the combination of therapies with different but complementary mechanisms of actions is now being considered by more and more drug developers as the best route to enhance efficiency, slow disease progression or even reversing NASH (Figure 2). Several drugs are currently being tested in combination with FXR agonists. The combinations of Semaglutide/Cilofexor/Firsocostat (Novo Nordisk and Gilead), Tropifexor/CVC (Allergan and Novartis) and Selonsertib/Cilofexor/Firsocostat (Gilead, now discontinued) have all been discussed in more detail above. In other drug combination studies, Novartis is recruiting $380 \mathrm{NASH}$ patients with stage 2 or $3 \mathrm{CRN}$ fibrosis to test Licogliflozin, an SGLT1/2 inhibitor, and Tropifexor alone or in combination, in a Phase 2 ELIVATE clinical study (ClinicalTrials.gov NCT04065841), which is expected to complete in 2023. Metacrine Inc is evaluating another FXR agonist, MET409, in a Phase 2a combination trial (ClinicalTrials. gov NCT04702490) with Empagliflozin (Jardiance), a SGLT2 inhibitor, in patients with T2D and NASH. Results are expected in the fourth quarter of 2021. Pfizer Inc has considered combination therapies as well and assessed the effect of PF-05221304 (an ACC inhibitor) and PF-06865571 (a DGAT2 inhibitor) on whole-liver fat in subjects with NAFLD compared to placebo in a short, 6-week Phase 2a clinical trial (ClinicalTrials.gov NCT03776175). Both drugs alone and in combination significantly reduced liver fat when compared to placebo, but unfortunately, there was no evidence of additivity (or synergy) with no significant differences between each monotherapy, when compared with the combination arm. At the end of 2020, Pfizer communicated that PF05221304 was no longer in its pipeline and it was discontinued as a monotherapy. Furthermore, Pfizer has recently included a Phase 1 clinical trial in their pipeline to evaluate PF-06865571 in combination with PF-06882961 (Danuglipron), a GLP-1R agonist, for the treatment of NASH

Clinical trials involving monotherapies are still much higher in number than combination studies and are typically further in their clinical drug journey than most combination studies. However, we would predict that our growing understanding of the complexity of liver disease will almost certainly drive an increase in business development activity and the signing of more agreements between pharmaceutical companies eager to combine their, hopefully, complementary therapeutic agents.

\section{Conclusion}

Liver disease represents one of the greatest areas of unmet medical need in the developed world, with the lack of a reliable and user-friendly diagnostic and the absence of tailored drug therapies combining to drive a relentless increase in the number of patients globally. Clinical intervention is woefully inadequate and is still often limited to changes in life-style advice, a situation that has altered little in over 10 years. However, what has changed in the last decade is the development of numerous therapies in later stage clinical trials that could offer real hope, and soon, to patients struggling with metabolic liver diseases, such as NASH and NAFLD. The complex and heterogeneous nature of these diseases has generated a portfolio of drug classes (from small molecule inhibitors to antibodies), each being evaluated as the first approved therapy specific to NASH. Whilst one of the pharmaceutical companies described in this review may indeed be a winner in the race for the first universally accepted medication for $\mathrm{NASH}$ as a monotherapy, it is likely that this will simply be the end of the beginning rather than the beginning of the end for NASH, NAFLD and liver disease therapeutic options. The plethora of drug classes currently being evaluated and the possible benefits of combining agents with different and complementary mechanisms of action will keep this area of drug discovery active and productive for another 10 years at least. 


\section{Disclosure}

Caroline Barelle, Andrew J Porter, and Marina Kovaleva are employees of Elasmogen Ltd. Soumya Palliyil is an employee of University of Aberdeen. Julia M Fraile reports being a Knowledge Transfer Partnership-Associate (Innovate UK) between the University of Aberdeen and Elasmogen Ltd, during the conduct of the study. Andrew J Porter reports personal fees from Elasmogen Ltd, during the conduct of the study. The authors report no other potential conflicts of interest in this work.

\section{References}

1. Chalasani N, Younossi Z, Lavine JE, et al. The diagnosis and management of nonalcoholic fatty liver disease: practice guidance from the American association for the study of liver diseases. Hepatology. 2018;67(1):328-357.

2. GlobalData. Non-alcoholic steatohepatitis (NASH) - opportunity analysis and forecasts to 2029; 2020. Available from: https://store.global data.com/report/gdhc113poa-non-alcoholic-steatohepatitis-nash-oppor tunity-analysis-and-forecasts-to-2029/. Accessed May 28, 2021.

3. Targher G, Byrne CD, Lonardo A, Zoppini G, Barbui C. Non-alcoholic fatty liver disease and risk of incident cardiovascular disease: a meta-analysis. J Hepatol. 2016;65(3):589-600. doi:10.1016/j. jhep.2016.05.013

4. Eslam M, Sanyal AJ, George J, International Consensus P. MAFLD: a consensus-driven proposed nomenclature for metabolic associated fatty liver disease. Gastroenterology. 2020;158(7):1999-2014e1991. doi:10.1053/j.gastro.2019.11.312

5. Ciardullo S, Perseghin G. Prevalence of NAFLD, MAFLD and associated advanced fibrosis in the contemporary United States population. Liver Int. 2021;41(6):1290-1293. doi:10.1111/liv.14828

6. Younossi ZM, Koenig AB, Abdelatif D, Fazel Y, Henry L, Wymer M. Global epidemiology of nonalcoholic fatty liver disease-metaanalytic assessment of prevalence, incidence, and outcomes. Hepatology. 2016;64(1):73-84. doi:10.1002/hep.28431

7. Lassailly G, Caiazzo R, Ntandja-Wandji LC, et al. Bariatric surgery provides long-term resolution of nonalcoholic steatohepatitis and regression of fibrosis. Gastroenterology. 2020;159(4):12901301e1295. doi:10.1053/j.gastro.2020.06.006

8. Smeuninx B, Boslem E, Febbraio MA. Current and future treatments in the fight against non-alcoholic fatty liver disease. Cancers (Basel). 2020;12(7):1714. doi:10.3390/cancers12071714

9. Kleiner DE, Brunt EM, Van Natta M, et al. Design and validation of a histological scoring system for nonalcoholic fatty liver disease. Hepatology. 2005;41(6):1313-1321. doi:10.1002/hep.20701

10. Omokaro SO, Golden JK. The regulatory state of nonalcoholic steatohepatitis and metabolism. Endocrinol Diabetes Metab. 2020;3(4): e00113. doi:10.1002/edm2.113

11. Sanderson L. PPARs: Important Regulators in Metabolism and Inflammation. Vol. 8. Dordrecht: Springer; 2010.

12. Gawrieh S, Noureddin M, Loo N, et al. Saroglitazar, a PPAR-alpha/ gamma agonist, for treatment of NAFLD: a randomized controlled double-blind phase 2 trial. Hepatology. 2021. doi:10.1002/hep.31843

13. Lefebvre P, Cariou B, Lien F, Kuipers F, Staels B. Role of bile acids and bile acid receptors in metabolic regulation. Physiol Rev. 2009;89 (1):147-191. doi:10.1152/physrev.00010.2008

14. Younossi ZM, Ratziu V, Loomba R, et al. Obeticholic acid for the treatment of non-alcoholic steatohepatitis: interim analysis from a multicentre, randomised, placebo-controlled phase 3 trial. Lancet. 2019;394(10215):2184-2196. doi:10.1016/S0140-6736(19) $33041-7$
15. Siddiqui MS, Van Natta ML, Connelly MA, et al. Impact of obeticholic acid on the lipoprotein profile in patients with non-alcoholic steatohepatitis. $J$ Hepatol. 2020;72(1):25-33. doi:10.1016/j. jhep.2019.10.006

16. Sinha RA, Bruinstroop E, Singh BK, Yen PM. Nonalcoholic fatty liver disease and hypercholesterolemia: roles of thyroid hormones, metabolites, and agonists. Thyroid. 2019;29(9):1173-1191. doi:10.1089/thy.2018.0664

17. Harrison SA, Bashir MR, Guy CD, et al. Resmetirom (MGL-3196) for the treatment of non-alcoholic steatohepatitis: a multicentre, randomised, double-blind, placebo-controlled, phase 2 trial. Lancet. 2019;394(10213):2012-2024. doi:10.1016/S0140-6736(19)32517-6

18. Marra F, Tacke F. Roles for chemokines in liver disease. Gastroenterology. 2014;147(3):577-594e571. doi:10.1053/j.gastro.2014.06.043

19. Ratziu V, Sanyal A, Harrison SA, et al. Cenicriviroc treatment for adults with nonalcoholic steatohepatitis and fibrosis: final analysis of the phase 2b CENTAUR Study. Hepatology. 2020;72(3):892-905. doi:10.1002/hep.31108

20. Anstee QM, Neuschwander-Tetri BA, Wong VW, et al. Cenicriviroc for the treatment of liver fibrosis in adults with nonalcoholic steatohepatitis: AURORA phase 3 Study design. Contemp Clin Trials. 2020;89:105922. doi:10.1016/j.cct.2019.105922

21. Pedrosa M, Seyedkazemi S, Francque S, et al. A randomized, double-blind, multicenter, phase $2 \mathrm{~b}$ study to evaluate the safety and efficacy of a combination of tropifexor and cenicriviroc in patients with nonalcoholic steatohepatitis and liver fibrosis: study design of the TANDEM trial. Contemp Clin Trials. 2020;88:105889. doi:10.1016/j.cct.2019.105889

22. Miyazaki M, Flowers MT, Sampath H, et al. Hepatic stearoyl-CoA desaturase-1 deficiency protects mice from carbohydrate-induced adiposity and hepatic steatosis. Cell Metab. 2007;6(6):484-496. doi:10.1016/j.cmet.2007.10.014

23. Iruarrizaga-Lejarreta M, Varela-Rey M, Fernandez-Ramos D, et al. Role of aramchol in steatohepatitis and fibrosis in mice. Hepatol Commun. 2017;1(9):911-927. doi:10.1002/hep4.1107

24. Di Lella S, Sundblad V, Cerliani JP, et al. When galectins recognize glycans: from biochemistry to physiology and back again. Biochemistry. 2011;50(37):7842-7857. doi:10.1021/bi201121m

25. Chalasani N, Abdelmalek MF, Garcia-Tsao G, et al. Effects of belapectin, an inhibitor of galectin-3, in patients with nonalcoholic steatohepatitis with cirrhosis and portal hypertension. Gastroenterology. 2020;158(5):1334-1345e1335. doi:10.1053/j.gastro.2019.11.296

26. Colca JR, McDonald WG, McCommis KS, Finck BN. Treating fatty liver disease by modulating mitochondrial pyruvate metabolism. Hepatol Commun. 2017;1(3):193-197. doi:10.1002/hep4.1036

27. Tillman EJ, Rolph T. FGF21: an emerging therapeutic target for non-alcoholic steatohepatitis and related metabolic diseases. Front Endocrinol (Lausanne). 2020;11:601290.

28. Harrison SA, Ruane PJ, Freilich BL, et al. Efruxifermin in non-alcoholic steatohepatitis: a randomized, double-blind, placebo-controlled, phase 2a trial. Nat Med. 2021;27(7):1262-1271. doi:10.1038/s41591-021-01425-3

29. Granata R. Peripheral activities of growth hormone-releasing hormone. J Endocrinol Invest. 2016;39(7):721-727. doi:10.1007/ s40618-016-0440-X

30. Stanley TL, Fourman LT, Feldpausch MN, et al. Effects of tesamorelin on non-alcoholic fatty liver disease in HIV: a randomised, double-blind, multicentre trial. Lancet HIV. 2019;6(12):e821-e830. doi:10.1016/S2352-3018(19)30338-8

31. Dharmalingam M, Yamasandhi PG. Nonalcoholic fatty liver disease and type 2 diabetes mellitus. Indian J Endocrinol Metab. 2018;22 (3):421-428. doi:10.4103/ijem.IJEM_585_17

32. Bril F, Biernacki DM, Kalavalapalli S, et al. Role of vitamin E for nonalcoholic steatohepatitis in patients with type 2 diabetes: a randomized controlled trial. Diabetes Care. 2019;42 (8):1481-1488. doi:10.2337/dc19-0167 
33. Sanyal AJ, Chalasani N, Kowdley KV, et al. Pioglitazone, vitamin E, or placebo for nonalcoholic steatohepatitis. $N$ Engl J Med. 2010;362 (18):1675-1685. doi:10.1056/NEJMoa0907929

34. Sumida Y, Yoneda M, Tokushige K, et al. Antidiabetic therapy in the treatment of nonalcoholic steatohepatitis. Int J Mol Sci. 2020;21 (6):1907. doi:10.3390/ijms21061907

35. Newsome PN, Buchholtz K, Cusi K, et al. A placebo-controlled trial of subcutaneous semaglutide in nonalcoholic steatohepatitis. $N$ Engl $J$ Med. 2020;384(12):1113-24.

36. Harrison SA, Wong VW, Okanoue T, et al. Selonsertib for patients with bridging fibrosis or compensated cirrhosis due to NASH: results from randomized Phase III STELLAR trials. J Hepatol. 2020;73 (1):26-39. doi:10.1016/j.jhep.2020.02.027

37. Loomba R, Noureddin M, Kowdley KV, et al. Combination therapies including cilofexor and firsocostat for bridging fibrosis and cirrhosis attributable to NASH. Hepatology. 2021;73(2):625-643. doi:10.1002/ hep. 31622

38. Ratziu V, Harrison SA, Francque S, et al. Elafibranor, an agonist of the peroxisome proliferator-activated receptor-alpha and -delta, induces resolution of nonalcoholic steatohepatitis without fibrosis worsening. Gastroenterology. 2016;150(5):1147-1159e1145. doi:10.1053/j.gastro. 2016.01.038

39. Perepelyuk M, Terajima M, Wang AY, et al. Hepatic stellate cells and portal fibroblasts are the major cellular sources of collagens and lysyl oxidases in normal liver and early after injury. Am $J$ Physiol Gastrointest Liver Physiol. 2013;304(6):G605-614. doi:10.1152/ ajpgi.00222.2012

40. Liu SB, Ikenaga N, Peng ZW, et al. Lysyl oxidase activity contributes to collagen stabilization during liver fibrosis progression and limits spontaneous fibrosis reversal in mice. FASEB J. 2016;30 (4):1599-1609. doi:10.1096/fj.14-268425
41. Harrison SA, Abdelmalek MF, Caldwell S, et al. Simtuzumab is ineffective for patients with bridging fibrosis or compensated cirrhosis caused by nonalcoholic steatohepatitis. Gastroenterology. 2018;155(4):1140-1153. doi:10.1053/j.gastro.2018.07.006

42. Dong ZL, Lin J, Lin J. Structural basis of assembly of the human T cell receptor-CD3 complex. Nature. 2019;573(7775):546-552. doi:10.1038/s41586-019-1537-0

43. Baruch A, Wong C, Chinn LW, et al. Antibody-mediated activation of the FGFR1/Klothobeta complex corrects metabolic dysfunction and alters food preference in obese humans. Proc Natl Acad Sci U S A. 2020;117(46):28992-29000. doi:10.1073/pnas.2012073117

44. Segal-Salto M, Barashi N, Katav A, et al. A blocking monoclonal antibody to CCL24 alleviates liver fibrosis and inflammation in experimental models of liver damage. JHEP Rep. 2020;2 (1):100064. doi:10.1016/j.jhepr.2019.100064

45. Mallat A, Teixeira-Clerc F, Lotersztajn S. Cannabinoid signaling and liver therapeutics. J Hepatol. 2013;59(4):891-896. doi:10.1016/j. jhep.2013.03.032

46. Neuschwander-Tetri BA. Therapeutic landscape for NAFLD in 2020. Gastroenterology. 2020;158(7):1984-1998e1983. doi:10.1053/j. gastro.2020.01.051

47. Dufour JF, Caussy C, Loomba R. Combination therapy for non-alcoholic steatohepatitis: rationale, opportunities and challenges. Gut. 2020;69(10):1877-1884. doi:10.1136/gutjnl-2019319104
Drug Design, Development and Therapy

\section{Publish your work in this journal}

Drug Design, Development and Therapy is an international, peerreviewed open-access journal that spans the spectrum of drug design and development through to clinical applications. Clinical outcomes, patient safety, and programs for the development and effective, safe, and sustained use of medicines are a feature of the journal, which has also

\section{Dovepress}

been accepted for indexing on PubMed Central. The manuscript management system is completely online and includes a very quick and fair peer-review system, which is all easy to use. Visit http://www. dovepress.com/testimonials.php to read real quotes from published authors. 